\title{
Electrophysiological effect of rotigaptide in rabbits with heart failure
}

\author{
Yu Liu, Haitao Li, Wenfang Xia, Shengbo Yu, Congxin Huang, He Huang
}

Department of Cardiology, Renmin Hospital of Wuhan University, Wuhan 430060, China

Submitted: 23 November 2011

Accepted: 11 March 2012

Arch Med Sci 2014; 10, 2: 374-380

DOI: $10.5114 /$ aoms.2012.31385

Copyright @ 2014 Termedia \& Banach

\section{Abstract}

Introduction: Rotigaptide is a new anti-arrhythmic peptide, which has recently been found to increase junctional conductance and prevent ischemia-induced ventricular tachycardia. In this study, we attempted to investigate the effects and mechanisms of rotigaptide on the vulnerability to ventricular arrhythmias in rabbits with heart failure (HF).

Material and methods: Chronic volume-pressure overload was used to induce HF. After rotigaptide infusion, an electrophysiological study was performed to record monophasic action potential (MAP), determine the effective refractory period (ERP) and ventricular fibrillation threshold (VFT), and assess the susceptibility to ventricular arrhythmia. Finally, real-time PCR was used to detect the changes of connexin 43 (Cx43) mRNA expression.

Results: $\mathrm{HF}$ rabbits exhibited significant down-regulation of $\mathrm{Cx} 43 \mathrm{mRNA}$, increase of effective refractory period (ERP) and decrease of VFT ( $p<0.05$, respectively). These changes resulted in an increase of vulnerability to ventricular tachyarrhythmias (VT/VF). Rotigaptide administration shortened ERP (113.3 $\pm 8.6 \mathrm{~ms}$ vs. $131.7 \pm 12.5 \mathrm{~ms}$, $p<0.05)$, restored VFT $(15.0 \pm 2.0$ V vs. $6.3 \pm 1.4 \mathrm{~V}, p<0.05)$, and decreased the vulnerability to VT/VF. However, short-term rotigaptide treatment had no significant effect on MAP duration (MAP duration at $90 \%$ repolarization: $169.3 \pm 6.0 \mathrm{~ms}$ vs. $172.7 \pm 6.2 \mathrm{~ms}, p>0.05)$ or connexin $43 \mathrm{mRNA}$ expression $(p>0.05)$.

Conclusions: Rotigaptide decreases the ERP, elevates VFT, and reduces the vulnerability to ventricular arrhythmias without changing $C \times 43$ expression in rabbits with HF. It may be a promising antiarrhythmic drug for preventing ventricular arrhythmia in HF.

Key words: rotigaptide, heart failure, connexin 43, ventricular arrhythmias.

\section{Introduction}

Heart failure (HF) is a major public health problem associated with a significant risk of morbidity and mortality. Patients with HF frequently manifest complex ventricular arrhythmias. Ventricular tachyarrhythmias are the most common arrhythmias associated with unexpected sudden cardiac death, the leading cause of death in these patients [1].

Gap junctions are low resistance connections between adjacent myocardial cells, consisting of proteins, most predominantly connexin 43 (Cx43), which form intercellular communications that are crucial for myocardial conduction [2]. Evidence has shown that disruption of gap junction coupling during pathological conditions plays a key role in arrhythmogenesis [3, 4].

Rotigaptide, a new anti-arrhythmic peptide, was recently found to increase junctional conductance and prevent ventricular tachycardia in a ca-
Corresponding author: Prof. He Huang Department of Cardiology Renmin Hospital of Wuhan University No. 238 Jiefang Road Wuchang District Wuhan 430060, China Phone: 86-27-88041911 E-mail: huangwhu2011@163.com 
nine ventricular ischemia model [5]. Rotigaptide has also been reported to reduce gap junction closing during acidosis [6], and to prevent intercellular uncoupling, which causes conduction velocity slowing during metabolic stress [7]. Abnormalities in connexin expression and function have also been reported in $\mathrm{HF}$, which may create serious arrhythmogenic substrate [8-10]. Given the critical role of connexin abnormalities in arrhythmogenesis, we hypothesized that gap junction coupling promoters such as rotigaptide may reduce ventricular arrhythmias in HF.

\section{Material and methods}

\section{Animal preparation}

Studies were performed on healthy adult New Zealand White rabbits of either sex with an initial body weight of $2.0-3.0 \mathrm{~kg}$. HF was produced by combined aortic insufficiency and aortic constriction in 2 sequential surgical procedures as described previously [11]. In brief, the animals were anesthetized with $3 \%$ pentobarbital sodium (30 mg/kg). Induction of aortic insufficiency was performed by inserting a catheter via the left carotid artery and repeatedly pushing it through the aortic valve to increase pulse pressure by at least $50 \%$. The severity of aortic insufficiency was assessed by 2D echocardiography with color flow mapping. Two weeks after aortic insufficiency, under general anesthesia with $3 \%$ pentobarbital sodium $(30 \mathrm{mg} / \mathrm{kg})$, the abdomen was opened, and the abdominal aorta was surgically dissected at a site slightly above the renal arteries. A catheter was then placed along the side of the isolated aorta segment. Thereafter, a suture was tightly tied around the aorta and the overlying catheter. The catheter was then pulled out, thus producing an approximately $50 \%$ aortic constriction above the renal arteries. Sham-operated animals underwent the same surgical procedure without aortic insufficiency and aortic constriction.

All experimental procedures were performed in accordance with the Guide for the Care and Use of Laboratory Animals published by the US National Institutes of Health (NIH Publication no. 85-23, revised 1996), and the protocol was approved by the Institutional Animal Care Committee from Wuhan University, People's Republic of China.

\section{Echocardiography measurements}

Left ventricular (LV) function was assessed by echocardiography (8 $\mathrm{MHz}$ probe, GE, VIVID7, USA). The anterior chest area was shaved, and two-dimensional images and $\mathrm{M}$-mode tracings were recorded. The LV end-diastolic dimension (LVEDD) and endsystolic dimension (LVESD) were measured. LV enddiastolic volume (LVEDV) and end-systolic volume (LVESV) were calculated using the simplified Simp- son's rule. LV ejection fraction (LVEF) and fractional shortening (LVFS) were then calculated using the following formulae: $\operatorname{LVEF}=\left[\left(\mathrm{LVEDD}^{3}-\mathrm{LVESD}^{3}\right) /\right.$ LVEDD $\left.^{3}\right] \times 100 \%$, LVFS $=[($ LVEDD - LVESD $) /$ LVEDD $]$ $\times 100 \%$.

\section{Drug administration}

The rabbits with HF were randomly assigned to receive either rotigaptide or saline, while the shamoperated rabbits were administered saline. Rotigaptide (Ac-d-Tyr-d-Pro-d-Hyp-Gly-d-Ala-Gly- $\mathrm{NH}_{2}$ ) was synthesized by Chinese Peptide Co., Hangzhou, Zhejiang, China. In the present study, rotigaptide was administered as an intravenous bolus loading dose $(1.5 \mu \mathrm{g} / \mathrm{kg})$ followed by a maintenance intravenous infusion ( $94 \mathrm{ng} / \mathrm{kg} / \mathrm{min}$ ) by a pump. The protocol of saline administration was similar to rotigaptide administration. All measurements were performed $20 \mathrm{~min}$ after the start of the infusion.

\section{Electrophysiological study in vivo}

On the day of the study, the animals were anesthetized with $3 \%$ pentobarbital sodium ( $30 \mathrm{mg} / \mathrm{kg}$ ), and ventilated with $\mathrm{O}_{2} 100 \%$. A median thoracotomy was performed, and the heart was exposed in a pericardial cradle. In order to electrically stimulate the heart, a slower intrinsic heart rate was needed. Thus the sinoatrial node was destroyed by injection of $0.1-0.3 \mathrm{ml}$ of $100 \mathrm{ml} / \mathrm{l}$ formaldehyde into the region between the right atrial appendage and superior vena cava. Continual epicardium bipolar pacing was delivered from the high right atrium at a cycle length of $300 \mathrm{~ms}$.

The technique of recording monophasic action potential (MAP) has been described in our previous study [12]. In brief, a custom-made MAP electrode was used. The MAP electrode penetrated into the epicardium of the left ventricular free wall in each group and the reference electrode was placed on the thorax. All signals were recorded with a polygraph (LEAD2000B, Jinjiang Ltd) and were filtered to record frequencies between $0.05 \mathrm{~Hz}$ and $30 \mathrm{~Hz}$ for the MAP.

Programmed electrical stimulation protocols were used to assess the susceptibility to ventricular arrhythmia. The stimulation protocol is characterized by two following stimuli (S1-S2) of variable basal cycle lengths and coupling intervals. The end point of testing was the induction of at least six consecutive non-driven ventricular responses after the extrastimuli application or completion of the stimulation protocol. A preparation was considered noninducible when programmed electrical stimulation produced either no ventricular premature beats or only self-terminating salvos of $<6$ beats. Stimuli were delivered using a pair of bipolar electrodes placed at the center of the anterior LV free wall. 
Extrastimuli of twice the diastolic threshold intensity and $2 \mathrm{~ms}$ in duration were delivered after 20 beats of ventricular drive with a coupling interval decreasing in steps of $5 \mathrm{~ms}$ until the ventricular effective refractory period (ERP) was reached. The ERP was defined as the longest S1S2 interval at which S2 failed to produce a propagated ventricular response. The interval between two drives was $1 \mathrm{~min}$. The stimulation procedures were repeated at four different basal cycle lengths (300 ms, 250 ms, $200 \mathrm{~ms}, 180 \mathrm{~ms}$ ). Occurrence of inducible arrhythmias was documented.

Finally, the ventricular fibrillation threshold (VFT) was determined. Bipolar electrodes were penetrated into the left ventricular apex. Electric stimulation was supplied at a stable paced cycle length of $100 \mathrm{~ms}$. The interval between each episode of stimulation was $1 \mathrm{~min}$. The initial pacing voltage was $2 \mathrm{~V}$, and progressively increased by a step of $1 \mathrm{~V}$. The VFT was defined as the smallest amount of voltage required to elicit ventricular fibrillation.

\section{Real-time polymerase chain reaction}

Total RNA was extracted from the LV free wall using Trizol reagent (Invitrogen, USA) according to the manufacturer's protocol. The expression levels of candidate genes were measured by real-time quantitative RT-PCR using a SYBR Green PCR Master mix (Biosystems). cDNA was amplified under the following conditions: $95^{\circ} \mathrm{C}$ for 2 min and then ran for 40 cycles at $95^{\circ} \mathrm{C}$ for $15 \mathrm{~s}, 59^{\circ} \mathrm{C}$ for $15 \mathrm{~s}$ and $72^{\circ} \mathrm{C}$ for $45 \mathrm{~s}$. The amplifications were carried out using the following primers: Cx43 forward primer 5'-AAGAGACCCCTGCCCACAT-3', reverse primer 5'-AGAGACACCAACGACACCACC-3'; $\beta$-actin forward primer 5'-CGAGA- TCGTGCGGGACAT-3', reverse primer 5'CAGGAA- GGAGGGCTGGAAC-3'. Amplification products of $C \times 43$ and $\beta$-actin were predicted to be $99 \mathrm{bp}$ and $184 \mathrm{bp}$, respectively. The mRNA levels of each gene were calculated using the $2-\Delta \Delta C T$ method [13].

\section{Statistical analysis}

All values are expressed as mean \pm standard deviation. T-test was used for between-group comparisons. One way ANOVA was used to compare more than two groups. Fisher's exact probability test was used to assess the inducibility of ventricular arrhythmia. A value of $p<0.05$ was considered statistically significant.

\section{Results}

\section{Heart failure parameters}

Table I shows characteristics of control rabbits and rabbits with HF. The increase in relative heart weight, LVEDD, LVESD, LVEDV and LVESV and the decrease of LVEF and LVFS indicates the presence of HF in comparison with control animals. Two rabbits in the HF group died during the electrophysiological experiment. Therefore, we collected and analyzed the following experimental data from 6 animals in each group.

\section{Electrophysiological variables}

Representative MAP records in a control, a HF and a $\mathrm{HF}+$ rotigaptide rabbit are shown in Figure $1 \mathrm{~A}$. Figure $1 \mathrm{~B}$ shows the MAP duration at $90 \%$ repolarization $\left(\mathrm{MAPD}_{90}\right)(\mathrm{CL}=300 \mathrm{~ms})$ in all 3 groups. $\mathrm{HF}$ resulted in significant $\mathrm{MAPD}_{90}$ prolongation, $p<0.05$. Rotigaptide had no significant effect on $\mathrm{MAPD}_{90}$ in rabbits with $\mathrm{HF}, p>0.05$.

Figure 2 shows the effect of rotigaptide on ERP in all 3 groups. The ERP was significantly longer in the HF group compared with the control group. Rotigaptide administration significantly shortened ERP in rabbits with $\mathrm{HF}$.

Figure 3 shows the VFT in all 3 groups. Compared with the sham group, the HF rabbits exhibited a marked decrease of VFT, $p<0.05$. Rotigaptide induced a significant increase of VFT in rabbits with HF, $p<0.05$.

Table I. Heart failure parameters

\begin{tabular}{|lccc|}
\hline Index & Control group $(n=6)$ & HF group $(n=8)$ & HF + rotigaptide group $(n=6)$ \\
\hline BW $[\mathrm{kg}]$ & $3.5 \pm 0.1$ & $2.6 \pm 0.2^{*}$ & $2.8 \pm 0.2^{*}$ \\
\hline LVW $[\mathrm{g}]$ & $3.6 \pm 0.1$ & $6.2 \pm 0.3^{*}$ & $6.2 \pm 0.5^{*}$ \\
\hline LVW/BW $[\mathrm{g} / \mathrm{kg}]$ & $1.0 \pm 0.1$ & $2.4 \pm 0.2^{*}$ & $2.3 \pm 0.2^{*}$ \\
\hline LVEDD $[\mathrm{cm}]$ & $1.5 \pm 0.2$ & $2.3 \pm 0.2^{*}$ & $2.4 \pm 0.5^{*}$ \\
\hline LVESD [cm] & $0.9 \pm 0.1$ & $1.9 \pm 0.2^{*}$ & $1.9 \pm 0.4^{*}$ \\
\hline LVEDV [ml] & $6.0 \pm 1.7$ & $18.3 \pm 3.8^{*}$ & $22.0 \pm 9.9^{*}$ \\
\hline LVESV [ml] & $1.4 \pm 0.5$ & $10.8 \pm 2.3^{*}$ & $12.7 \pm 6.5^{*}$ \\
\hline LVEF [\%] & $75.3 \pm 4.1$ & $38.9 \pm 3.3^{*}$ & $38.4 \pm 6.9^{*}$ \\
\hline LV FS [\%] & $40.9 \pm 3.6$ & $17.4 \pm 2.5^{*}$ & $17.3 \pm 3.2^{*}$ \\
\hline
\end{tabular}

Values are mean $\pm S D$. HF - heart failure, BW - body weight, $L V W$ - left ventricle weight, LVEDD - left ventricular end-diastolic dimension, LVESD - left ventricular end-systolic dimension, LVEDV - left ventricular end-diastolic volume, LVESV - left ventricular end-systolic volume, LVEF - left ventricular ejection fraction, LVFS - left ventricular fractional shortening. Compared with control group, ${ }^{*} p<0.05$ 
A
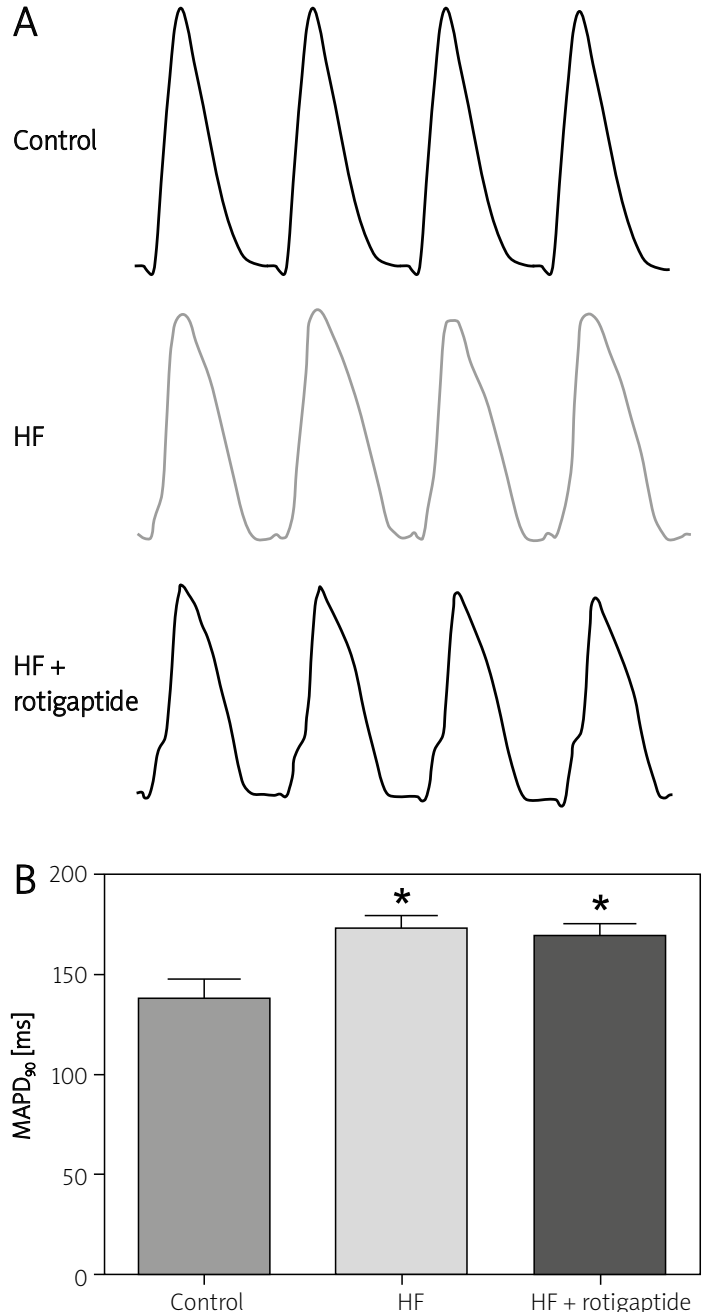

Figure 1. Effect of rotigaptide on MAPD in HF rabbits. A - Representative MAP records in a control, a HF and a $\mathrm{HF}+$ rotigaptide rabbit, respectively. $\mathrm{B}$ $\mathrm{MAPD}_{90}$ in all 3 groups. MAPD 90 was determined at a basic (S1-S1) cycle length of $300 \mathrm{~ms}$

Data are expressed as mean $\pm S D, n=6$ in each group. ${ }^{*} p<0.05$ vs. control group

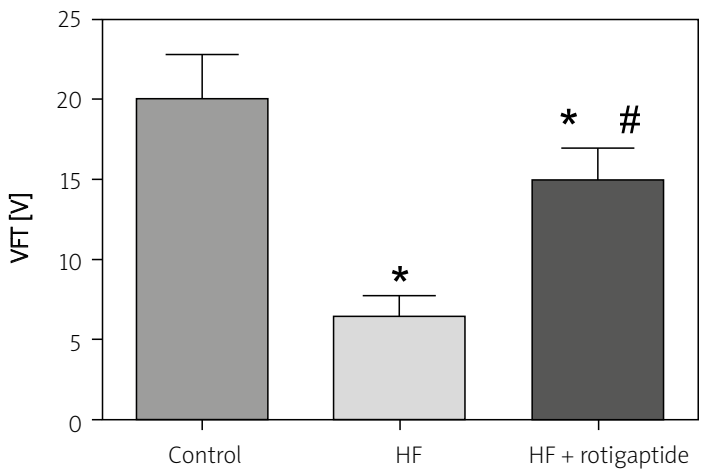

Figure 3. Effect of rotigaptide on VFT. Data are expressed as mean $\pm \mathrm{SD}, n=6$ in each group. $\mathrm{HF}$ resulted in a decrease of VFT, which was restored by rotigaptide administration

${ }^{*} p<0.05$ vs. control group. ${ }^{*} p<0.05$ vs. HF group
VT/VF induction was attempted by programmed stimulation in each of 6 rabbits from the 3 animal groups. In the control group, no VT/VF was induced. In the HF group, $3 \mathrm{VF}$ and $2 \mathrm{VT}$ (sustained $>30 \mathrm{~s}$ ) were induced (compared with control group, $p<0.05$ ). In the HF + rotigaptide group, only one sustained VT was induced (compared with control group, $p>0.05$ ).

\section{Cx43 expression}

Figure 4 shows the $C \times 43$ mRNA expression in all 3 groups. $\mathrm{C} \times 43$ mRNA expression in HF rabbits, compared with the control group, was significantly reduced by $60 \%, p<0.05$. Rotigaptide had no significant effect on Cx43 mRNA expression in rabbits with $\mathrm{HF}, p>0.05$.

\section{Discussion}

The main findings of the present study are that $\mathrm{HF}$ resulted in down-regulation of $\mathrm{Cx} 43$ expression, increase of ERP, decrease of VFT and increase of

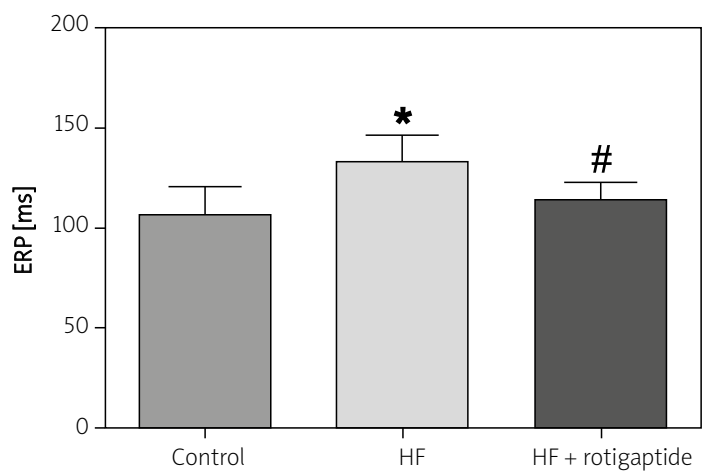

Figure 2. Effect of rotigaptide on ERP. ERP was determined at a basic (S1-S1) cycle length of $300 \mathrm{~ms}$. Data are expressed as mean $\pm \mathrm{SD}, n=6$ in each group. HF resulted in the prolongation of ERP, which was restored by rotigaptide administration ${ }^{*} p<0.05$ vs. control group. ${ }^{*} p<0.05$ vs. HF group

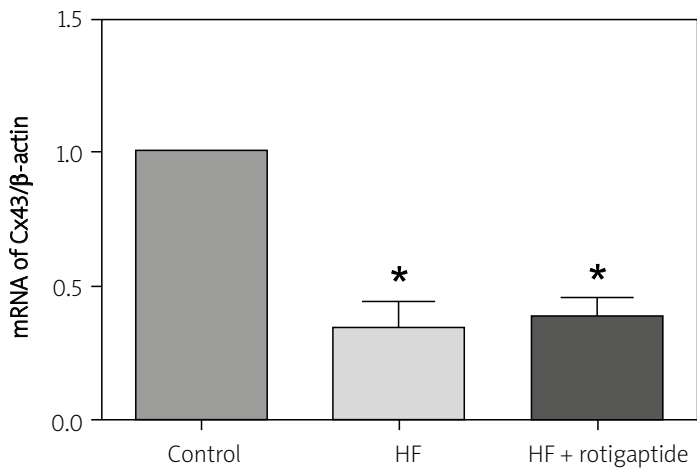

Figure 4. Real-time $P C R$ analysis of $C \times 43$ in all 3 groups. Data are expressed as mean $\pm \mathrm{SD}, n=6$ in each group. HF resulted in a decrease of $C \times 43$ mRNA expression. Rotigaptide had no significant effect on Cx43 mRNA expression in $\mathrm{HF}$ rabbits ${ }^{*} p<0.05$ vs. control group 
ventricular tachyarrhythmias. Rotigaptide administration sharply shortened ERP, increased VFT and reduced induction of ventricular tachyarrhythmias without changing $\mathrm{Cx} 43$ expression. Our findings underscore that rotigaptide is of use to reduce the vulnerability of rabbit hearts to ventricular arrhythmias after HF.

In the present study, we used pressure-volume overload to induce the HF model, which has been proved to be pro-arrhythmic [14]. The animals in the HF group showed an increase in LVEDD, LVESD, LVEDV and LVESV, and a decrease of LVEF and LVFS. Furthermore, body weight and the ratio of left ventricle weight to body weight were also increased. A possible reason for the significant difference of body weight between control and HF groups was malnutrition because food intake significantly decreased in the HF group in the later period of the HF model.

Gap junctions, assembled from connexins, form intercellular communications for the orderly spread of current flow required for the regular rhythm of the healthy heart. It is now well recognized that alterations in gap junctions and connexins have emerged as important factors to be considered in understanding the pro-arrhythmic substrate [15]. The most consistently observed alteration in ventricular Cx expression involves downregulation of Cx43 in a variety of models of HF. Disorganization of gap junction distribution and down-regulation of Cx43 has been shown in patients with dilated, ischemic, and inflammatory cardiomyopathies [16]. Downregulation of $\mathrm{C} \times 43$ was also demonstrated in several animal models. In a canine model of pacinginduced HF, Poelzing et al. [8] reported that reduced Cx43 expression in HF was accompanied by significant reductions in intercellular coupling between transmural muscle layers in association with marked dispersion of repolarization. In a rabbit model of HF induced by pressure-volume overload, Wiegerinck et al. [17] showed that heterogeneously reduced connexin 43 in the midmyocardium is associated with increased transmural dispersion in refractoriness and conduction, and with increased arrhythmia inducibility. Sato et al. [10] showed that alterations of $\mathrm{C} \times 43$ expression and phosphorylation in concert with interstitial fibrosis may create serious arrhythmogenic substrate through inhibition of cellto-cell coupling during the development of HF in the cardiomyopathic hamster. The present study using $\mathrm{HF}$ rabbits is in line with these studies in terms of Cx43 downregulation during the development of HF.

Rotigaptide is a novel and highly stable analogue of the naturally occurring antiarrhythmic peptide first described in 1980 [18]. Rotigaptide increases gap junction intercellular communication in ventricular cardiomyocytes without any effect on membrane currents [6], prevents acidosis-induced ventricular conduction slowing [5], and prevents and reverts atrial conduction slowing [7, 19]. In addition, rotiga- ptide treatment prevents spontaneous ventricular arrhythmias during myocardial ischemia/reperfusion injury [5], prevents reentrant ventricular tachycardia during myocardial ischemia [20], and delays the onset of ouabain-induced conduction block [21]. A previous study demonstrated that reentry is the underlying electrophysiological mechanism of ventricular tachyarrhythmias [22]. Gap junctional uncoupling causes conduction slowing and therefore provides circumstances leading to reentry, which may form an arrhythmogenic mechanism for ventricular tachyarrhythmias [4]. The study by Zhou et al. [23] showed that VFT was lower in HF dogs than control animals. In the present study, rotigaptide significantly decreased the vulnerability of rabbit hearts to ventricular arrhythmias after HF. Our present study also found that HF resulted in a decrease of VFT, and this decrease was ameliorated by rotigaptide treatment. In a recent report by Li et al. [24], rotigaptide treatment decreased the defibrillation threshold and improved the rate of successful defibrillation in a swine model. This finding may provide a mechanistic explanation for the antiarrhythmic effect of rotigaptide in HF.

A study showed that 1-5 $\mathrm{h}$ rotigaptide treatment had no effect on Cx43 expression in neonatal cardiomyocytes or HeLa cells [25]. In another study, rotigaptide increased connexin 43 expression after $24 \mathrm{~h}$ in cultured rat neonatal cardiomyocytes, but such an effect was unlikely to explain changes after short-term intravenous administration [26]. In our present study, we also found that rotigaptide did not affect Cx43 mRNA expression after short-term administration (within $2 \mathrm{~h}$ ). These studies suggested that rotigaptide may increase intercellular coupling without affecting gap junction expression after shortterm administration, even in the presence of significant gap junction down-regulation. This may be the main mechanism for reducing the vulnerability of rabbit hearts to ventricular arrhythmias after HF.

In a rabbit model of cardiac arrest, Zhong et al. found that rotigaptide significantly decreased defibrillation voltage requirements but had no effect on heart rate, QRS duration, QT interval, ventricular ERP, MAP duration (MAPD) or on Cx43 density [27]. However, other studies showed that rotigaptide treatment shortened ERP in animal models of infarction and HF, respectively $[28,29]$. In our present study, we also found that rotigaptide treatment significantly shortened ventricular ERP. We think the discrepancy among these studies may result from different experimental models. Rotigaptide may shorten ERP in animals with prolonged ERP through enhancing intercellular electrical coupling. Action potential duration was determined by ionic currents. A previous study proved that rotigaptide had no significant effect on membrane currents and no effect on cellular repolarization [21]. This may explain why rotigaptide had no significant effect on MAPD in heart failure rabbits in our study. 
Rotigaptide increases gap junction intercellular communication in ventricular cardiomyocytes. However, the mechanisms by which rotigaptide alters connexin function remain unclear. Theoretically, rotigaptide could increase gap junction intercellular communication by affecting connexin expression, connexin gating, and connexin distribution. It has been reported that rotigaptide increases the activity of protein kinase $C$ and increases the phosphorylation of Cx43 [30]. Phosphorylation of the C terminal tail of $\mathrm{Cx} 43$ is an important regulator of connexin gating [31]. Axelsen et al. [32] reported that rotigaptide site-specifically suppresses dephosphorylation of serine residues in the C-terminal tail of $\mathrm{Cx} 43$ in rat hearts subjected to ischemia. However, another study failed to observe changes in the phosphorylation status of connexin 43 in response to rotigaptide [25]. To ultimately determine whether rotigaptide specifically modulates the phosphorylation status of $\mathrm{C} \times 43$ on any of the multiple phosphorylation sites of the carboxyl terminus may require other techniques such as mass spectrometry, which would help to elucidate a concise mechanism of action of this peptide. Another potential, yet unexplored, mechanism of action of rotigaptide action is the effect it may have on $\mathrm{Cx} 43$ hemichannel function.

There are several limitations in this study. First, we did not examine the plasma concentrations of rotigaptide or perform dose-response studies. Several previous studies have evaluated the dose response effect of rotigaptide after bolus plus infusion intravenous dosing. These studies indicated that lower doses and concentrations of rotigaptide may have had similar effects $[5,27]$. Second, we did not measure conduction velocity and intercellular electrical coupling owing to technical reasons. Previous studies have demonstrated that rotigaptide treatment can increase ventricular gap junction coupling and improve conduction $[5,33]$. Optical mapping and double-cell patch voltage clamp studies could be the next step to better understand how this drug exerts its antiarrhythmic effect in HF. Third, transmural electrophysiological heterogeneities are known to be an important substrate of arrhythmogenesis in HF [34]. In this study, we did not examine the transmural heterogeneities of cellular repolarization and their potential role in HF-related arrhythmias.

In conclusion, our study suggests that the new anti-arrhythmic peptide rotigaptide reduces the vulnerability to ventricular arrhythmias and increases the VFT in rabbits with HF. Rotigaptide may be a promising antiarrhythmic drug for use in preventing ventricular arrhythmia in HF.

\section{Acknowledgments}

This work was supported by grants from the National Natural Science foundation of China (No. 30700312, No. 81070142 and No. 81100129).

\section{References}

1. Tomaselli GF, Zipes DP. What causes sudden death in heart failure? Circ Res 2004; 95: 754-63.

2. Kanno S, Saffitz JE. The role of myocardial gap junctions in electrical conduction and arrhythmogenesis. Cardiovasc Pathol 2001; 10: 169-77.

3. De Groot JR, Coronel R. Acute ischemia-induced gap junctional uncoupling and arrhythmogenesis. Cardiovasc Res 2004; 62: 323-34.

4. Cabo C, Boyden PA. Heterogeneous gap junction remodeling stabilizes reentrant circuits in the epicardial border zone of the healing canine infarct: a computational study. Am J Physiol Heart Circ Physiol 2006; 291: H2606-16.

5. Xing D, Kjølbye AL, Nielsen MS, et al. ZP123 increases gap junctional conductance and prevents reentrant ventricular tachycardia during myocardial ischemia in open chest dogs. J Cardiovasc Electrophysiol 2003; 14: 510-20.

6. Eloff BC, Gilat E, Wan X, Rosenbaum DS. Pharmacological modulation of cardiac gap junctions to enhance cardiac conduction: evidence supporting a novel target for antiarrhythmic therapy. Circulation 2003; 108: 3157-63.

7. Haugan K, Olsen KB, Hartvig L, et al. The antiarrhythmic peptide analog ZP123 prevents atrial conduction slowing during metabolic stress. J Cardiovasc Electrophysiol 2005; 16: 537-45.

8. Poelzing S, Rosenbaum DS. Altered connexin43 expression produces arrhythmia substrate in heart failure. Am J Physiol Heart Circ Physiol 2004; 287: H1762-70.

9. Ai X, Pogwizd SM. Connexin 43 downregulation and dephosphorylation in nonischemic heart failure is associated with enhanced colocalized protein phosphatase type 2A. Circ Res 2005; 96: 54-63.

10. Sato T, Ohkusa T, Honjo H, et al. Altered expression of connexin43 contributes to the arrhythmogenic substrate during the development of heart failure in cardiomyopathic hamster. Am J Physiol Heart Circ Physiol 2008; 294: H1164-73.

11. Liu Y, Huang H, Xia W, et al. Inhibition of NADPH oxidase up-regulates connexin 43 and ameliorates electrical remodeling in rabbits with heart failure. Biomed Aging Pathol 2011; 1: 33-8..

12. Huang C, Bao M, Jiang H, Liu J, Yang B, Wang T. Differences in the changing trends of monophasic action potential duration and effective refractory period of the ventricular myocardium after myocardial infarction in vivo. Circ J 2004; 68: 1205-09.

13. Liu Y, Huang H, Xia W, Tang Y, Li H, Huang C. NADPH oxidase inhibition ameliorates cardiac dysfunction in rabbits with heart failure. Mol Cell Biochem 2010; 343: 143-53.

14. Pogwizd SM Nonreentrant mechanisms underlying spontaneous ventricular arrhythmias in a model of nonischemic heart failure in rabbits. Circulation 1995; 92: 1034-48.

15. Takamatsu T. Arrhythmogenic substrates in myocardial infarct. Pathol Int 2008; 58: 533-43.

16. Kostin S, Rieger M, Dammer S, et al. Gap junction remodeling and altered connexin43 expression in the failing human heart. Mol Cell Biochem 2003; 242: 135-44.

17. Wiegerinck RF, van Veen TA, Belterman CN, et al. Transmural dispersion of refractoriness and conduction velocity is associated with heterogeneously reduced connexin43 in a rabbit model of heart failure. Heart Rhythm 2008; 5: 1178-85.

18. Kjølbye AL, Haugan K, Hennan JK, Petersen JS. Pharmacological modulation of gap junction function with the novel compound rotigaptide: a promising new principle for 
prevention of arrhythmias. Basic Clin Pharmacol Toxicol 2007; 101: 215-30.

19. Haugan K, Kjølbye AL, Hennan JK, Petersen JS. Rotigaptide (ZP123) reverts established atrial conduction velocity slowing. Cell Commun Adhes 2005; 12: 271-78.

20. Hennan JK, Swillo RE, Morgan GA, et al. Rotigaptide (ZP123) prevents spontaneous ventricular arrhythmias and reduces infarct size during myocardial ischemia/reperfusion injury in open-chest dogs. J Pharmacol Exp Ther 2006; 317: 236-43.

21. Kjølbye AL, Knudsen CB, Jepsen T, Larsen BD, Petersen JS. Pharmacological characterization of the new stable antiarrhythmic peptide analog Ac-D-Tyr-D-Pro-D-Hyp-Gly-D-AlaGly-NH2 (ZP123): in vivo and in vitro studies. J Pharmacol Exp Ther 2003; 306: 1191-9.

22. Boukens BJ, Christoffels VM, Coronel R, Moorman AF Developmental basis for electrophysiological heterogeneity in the ventricular and outflow tract myocardium as a substrate for life-threatening ventricular arrhythmias. Circ Res 2009; 104: 19-31.

23. Zhou SX, Lei J, Fang C, Zhang YL, Wang JF. Ventricular electrophysiology in congestive heart failure and its correlation with heart rate variability and baroreflex sensitivity: a canine model study. Europace 2009; 11: 245-51.

24. Li JS, Zhong JQ, Zeng QX, Liu HZ, Su GY, Zhang Y. Effect of ZP123, a gap junction modifier, on prolonged ventricular fibrillation in swine. Cardiology 2011; 118: 147-52.

25. Clarke TC, Thomas D, Petersen JS, Evans WH, Martin PE. The antiarrhythmic peptide rotigaptide (ZP123) increas es gap junction intercellular communication in cardiac myocytes and HeLa cells expressing connexin 43. Br I Pharmacol 2006; 147: 486-95.

26. Stahlhut M, Petersen JS, Hennan JK, Ramirez MT. The antiarrhythmic peptide rotigaptide (ZP123) increases connexin 43 protein expression in neonatal rat ventricular cardiomyocytes. Cell Commun Adhes 2006; 13: 21-7.

27. Zhong JQ, Laurent G, So PP, Hu X, Hennan JK, Dorian P. Effects of rotigaptide, a gap junction modifier, on defibrillation energy and resuscitation from cardiac arrest in rabbits. J Cardiovasc Pharmacol Ther 2007; 12: 69-77.

28. Macia E, Dolmatova E, Cabo C, et al. Characterization of gap junction remodeling in epicardial border zone of healing canine infarcts and electrophysiological effects of partial reversal by rotigaptide. Circ Arrhythm Electrophysiol 2011; 4: 344-51.

29. Wiegerinck RF, de Bakker JM, Opthof T, et al. The effect of enhanced gap junctional conductance on ventricular conduction in explanted hearts from patients with heart failure. Basic Res Cardiol 2009; 104: 321-32.

30. Dhein S, Larsen BD, Petersen JS, Mohr FW. Effects of the new antiarrhythmic peptide ZP123 on epicardial activation and repolarization pattern. Cell Commun Adhes 2003; 10: $371-8$.

31. Lampe PD, Lau AF. The effects of connexin phosphorylation on gap junctional communication. Int J Biochem Cell Biol 2004; 36: 1171-86.

32. Axelsen LN, Stahlhut M, Mohammed S, et al. Identification of ischemia-regulated phosphorylation sites in connexin43: a possible target for the antiarrhythmic peptide analogue rotigaptide (ZP123). J Mol Cell Cardiol 2006; 40: 790-8.

33. Lin X, Zemlin C, Hennan JK, Petersen JS, Veenstra RD. Enhancement of ventricular gap-junction coupling by rotigaptide. Cardiovasc Res 2008; 79: 416-26.

34. Akar FG, Rosenbaum DS. Transmural electrophysiological heterogeneities underlying arrhythmogenesis in heart failure. Circ Res 2003; 93: 638-45 